\title{
Manejo clínico de DIP / DIPA doença inflamatória pélvica e de repetição: uma revisão narrativa
}

\author{
Clinical management of DIP / DIPA pelvic inflammatory disease and recurrence: a \\ narrative review
}

Manejo clínico de la enfermedad inflamatoria pélvica DIP / DIPA y recurrencia: una revisión narrativa

Francisca Amanda de Souza Mesquita ${ }^{1 *}$, Adelaide Gomes Martins ${ }^{1}$, Sabrina Cabral Rego ${ }^{1}$, Paula Barros Pereira ${ }^{1}$.

\begin{abstract}
RESUMO
Objetivo: Revisar sobre o manejo clínico de doença inflamatória pélvica e de repetição (DIP/DIPA). Revisão Bibliográfica: A DIP é uma infecção que se origina no trato genital inferior e que em muitos momentos pode atingir os órgãos do trato genital superior feminino, como o endométrio, tubas uterinas ou ovários. A maior prevalência de faixa etária é em mulheres que se encontram sexualmente ativas entre 15-24 anos de idade. É uma doença que se tratada no início consegue minimizar os prejuízos para a saúde da mulher. Se não tratada, a doença pode causar afetar o transporte dos óvulos e dos espermatozoides, acarretando dificuldades para a mulher engravidar ou contribui para chances de uma gestação ectópica. O tratamento da doença inicialmente é feito com o uso de antibióticos orais e intramusculares, caso a paciente apresente quadro de infecção, faz-se necessário a internação para uso de antibióticos endovenosos, e em alguns casos procedimento cirúrgico sendo preciso estabelecer repouso físico e sexual durante o tratamento. Considerações finais: Por se tratar de uma doença de clínica com variações, e em algumas situações assintomática, preconiza a dificuldade de se diagnosticar no início, para que tenha um tratamento adequado e eficiente a natureza da doença.
\end{abstract}

Palavras-chave: Doença inflamatória pélvica, Neisseria Gonorrhoeae, Tratamento.

\begin{abstract}
Objective: To review the clinical management of pelvic inflammatory and recurrent disease (DIP/DIPA). Bibliographic Review: DIP is an infection that originates in the lower genital tract and that in many moments can reach the female upper genital tract organs, such as the endometrium, fallopian tubes or ovaries. The highest prevalence of age group is in women who are sexually active between 15-24 years of age. It is a disease that if treated at the beginning manages to minimize the damage to the woman's health. If left untreated, the disease can affect the transport of eggs and sperm, causing difficulties for women to become pregnant or contributing to the chances of an ectopic pregnancy. The treatment of the disease is initially made with the use of oral and intramuscular antibiotics, if the patient has an infection, it is necessary to be hospitalized for the use of intravenous antibiotics, and in some cases, a surgical procedure requiring physical and sexual rest during treatment. Final considerations; As it is a clinical disease with variations, and in some cases asymptomatic, it recommends the difficulty of diagnosing it at the beginning, so that the nature of the disease is adequately and efficiently treated.
\end{abstract}

Keywords: Pelvic inflammatory disease, Neisseria Gonorrhoeae, Treatment.

1 Universidade Federal do Amazonas (UFAM), Manaus - AM. *E-mail: dra.amandamesquita@gmail.com 
RESUMEN

Objetivo: Revisar el manejo clínico de la enfermedad pélvica inflamatoria y recurrente (DIP/DIPA). Revisión bibliográfica: DIP es una infección que se origina en el tracto genital inferior y que en muchos momentos puede llegar a los órganos del tracto genital superior femenino, como el endometrio, las trompas de Falopio o los ovarios. La mayor prevalencia del grupo de edad se da en mujeres sexualmente activas entre los 15 y los 24 años. Es una enfermedad que si se trata al inicio consigue minimizar el daño a la salud de la mujer. Si no se trata, la enfermedad puede afectar el transporte de óvulos y espermatozoides, provocando dificultades para que las mujeres queden embarazadas o contribuyendo a las posibilidades de un embarazo ectópico. El tratamiento de la enfermedad se realiza inicialmente con el uso de antibióticos orales e intramusculares, si el paciente tiene una infección es necesario ser hospitalizado para el uso de antibióticos intravenosos, y en algunos casos, un procedimiento quirúrgico que requiera reposo físico y sexual durante Tratamiento Consideraciones finales: Al tratar-se dé una enfermedad clínica con variaciones, y en algunos casos asintomática, recomienda la dificultad de diagnosticarla al inicio, para que la naturaleza de la enfermedad sea tratada de manera adecuada y eficiente.

Palabras clave: Enfermedad pélvica inflamatoria, Neisseria Gonorrhoeae, Tratamiento.

\section{INTRODUÇÃO}

As dores abdominais agudas podem ser caracterizadas como atributo de dores resistentes e persistentes que fazem com que o paciente habitualmente procure auxílio no pronto-atendimento (SAMRAJ GP, et al., 2004).

A doença inflamatória pélvica (DIP) é uma doença que possui episódio inflamatório de caráter infecciosa e que pode abranger as estruturas do trato genital superior. É pré instituída, de modo geral, por acréscimo do agente infeccioso vaginal ou cervical, de forma natural ou em decorrência a processos como pode-se citar a colocação de dispositivo intrauterino (DIU), biópsia de endométrio e curetagem (BRASIL, 2006).

Caracterizada como inflamação aguda do trato genital superior feminino, possuindo capacidade de acometer o endométrio, trompas uterinas, ovários, peritônio e estruturas pélvicas adjacentes (MITCHELL C e PRABHU M, 2013). Já na visão de Romanelli RMC, et al. (2013), se dá por meio de processo inflamatório infeccioso que pode atingir algumas estruturas do trato genital superior feminino, como ovários, útero, tubas uterinas, e estruturas anexas, é vir a originar uma ooforite, salpingite, endometrite, abscesso tubo-ovariano e peritonite.

É uma doença que alcança uma faixa de um milhão de mulheres no período de um ano nos Estados Unidos, cujas causas são maiores em relação infertilidade, gravidez ectópica, dor pélvica crônica e outras categorias de alta morbimortalidade do sexo feminino (LAREAU SM e BEIGI RH, 2008). É a síndrome clínica que engloba as infecções agudas do trato genital superior feminino (IBARROLA M, et al., 2009).

Sweetr $L$ (2009), relata que a DIP resulta da contaminação via ascendente de microorganismos da vagina ou endocolo até ao endométrio, trompas de Falópio, ovários e/ou estruturas adjacentes e ainda segundo o autor levando sobretudo a endometrite e salpingite, mas podendo também ocorrer parametrite, ooforite e/ou abcesso tubo-ovárico.

Segundo Sousa FB, et al. (2018), a DIP é processo inflamatório de natureza polimicrobiana que pode causar o comprometimento do trato genital feminino. O que é causado pela alta carga de microrganismos do trato genital inferior, de forma espontânea ou apropriado a procedimentos como inserção de dispositivo intrauterino (DIU), biópsia de endométrio e curetagem. Seus principais agentes são patógenos e transmissíveis sexualmente, como Neisseria gonorrhoeae e Chlamydia trachomatis (NESS RB, 2005). Contudo, podem também ser responsáveis microrganismos da microbiota vaginal (anaeróbios, Gardnerellavaginalis, Haemophilusinfluenzae, bacilos Gram-negativos e Streptococcus agalactiae) (HODSON A, 2009). 
A infecção das trompas uterinas é o diagnóstico mais sério de concluir a DIP, o que pode vir ater implicação em uma fibrose que inibe a passagem dos óvulos ao útero, provavelmente ocasionando a esterilidade. Uma trompa uterina obstada pode cometer que um óvulo fertilizado seja disseminado na trompa ao invés do útero, muito conhecido como gravidez ectópica, que pode trazer risco a vida da mulher podendo chegar até a ruptura da trompa provocando hemorragia (TORTORA G, et al., 2005).

De acordo com Decherney AH, et al. (2003), a ocorrência da DIP pode acometer-se de três modos, sendo estes: Via linfática: muito comum pós-parto, pós- aborto e, em alguns momentos, após aplicação de DIU. provoca com uma certa frequência uma celulite parametrial. Outro modo de ocorrer e por via hematogénea: mais rara de ocorrer, mas que pode ser uma das vias na tuberculose (TB) genital. E por último a Via ascendente: é a mais constante, assim como a origem de endometrites, salpingites, salpingo-ooforites e peritonites. O intervalo de tempo entre a contaminação e o aparecimento dos sintomas vai geralmente de dois a quatro dias, mas pode chegar a um mês.

Em seu estudo Lareau SM e Beigi RH (2008), ressalta que a incidência da DIP é bem difícil de designar e estabelecer. Sua prevalência ainda é desvalorizada, pois a maioria dos casos é subclínica (> $60 \%)$, Já Judlin PG (2009), afirma que a real prevalência de DIPA é subestimada, mas Segundo o consenso é que tenha elevado seu crescimento no decorrer dos últimos anos, certamente em razão do diagnóstico mais frequente, por algumas mudanças nos hábitos das mulheres nos últimos anos, e ainda mediante a colocação do autor a maior prevalência de faixa etária é em mulheres sexualmente ativas entre 15-24 anos de idade.

Ainda segundo a colocação do autor acima citado eles abordam a doença inflamatória pélvica de repetição (DIPA), como uma situação que embasa-se em um conjunto de anormalidades que fica acometida na tuba uterina, contendo salpingite, piossalpinge e abscesso tubo-ovariano, como um saldo de infecção outrora de germes da vagina e colo uterino, sendo muito comum a Neisseria gonorrheae e Chlamydiatrachomatis, uma vez que esses dois patógenos são isolados em $12,2 \%$ dos casos.

Ainda segundo o relato do autor Judlin PG (2009), o quadro de DIPA se distingue ou é descoberto por ser deparado por dor no baixo ventre e por estar com elevado agrupamento no colo uterino e palpação dos anexos, podendo ou não vir com episódios de febre. O quadro clínico quando está em associação aos achados laboratoriais apresenta os valores precursores positivos de apenas $65 \%$ a $90 \%$, mesmo quando a realização é feita por profissionais capacitados e com experiência. É importante ressaltar que a parte epidemiológica e clínica, reafirma que além de graves complicações que a DIP pode acometer a mulher, fazse necessário que seja averiguada para DIP diante de sua dúvida pela histórico clinico ou por meio do exame ginecológico, não abordando a antecipação a procedimentos transcervicais (KHAN ZE, et al., 2006).

O diagnóstico clínico da DIP é duvidoso devido à suas diversas variáveis de sintomas sendo a dor geralmente é de característica bilateral e quando mensurado o tempo pode chegar a duas semanas. Outros sintomas que podem apresentar na DIP são dores anexais, dor à mobilização do colo e secreção purulenta endocervical. Dor e macicez em hipocôndrio direito costuma ser acompanhado de infecção por Neisseria gonorrhoeae e Chlamydia trachomatis, podendo evoluir com acometimento hepático (SCHNEE DM, 2009).

Alguns pacientes com DIP apresentam sangramento uterino anormal. Outros sintomas inespecíficos como uretrite, proctite, febre e calafrios também podem ser observados. Alguns pacientes desenvolvem DIP sem apresentar nenhuma manifestação clínica (ROSS J, et al., 2010).

O corrimento vaginal secundário a endometrite, cervicite ou vaginose pode não se caracterizar de maneira particular, mas quando é verificado sua ausência, pode-se considerar um alto valor preditivo para o negativo. Algumas outras inquietações clínicas que pode dar indicações de diagnóstico são: dor abdominal baixa (na maioria das vezes bilateral), febre $\left(38^{\circ} \mathrm{C}\right)$, sangramento que não esperados (como metrorragia), disúria, dispaureunia, princípio de dor em associação à menstruação, além de períodos apresentar náuseas e vômitos (MANAVI K, 2006). Quando o quadro clínico traz algum indicativo e não tem existência de outro diagnóstico possível, faz-se necessário dar início ao tratamento para minimizar o predomínio de possíveis sequelas reprodutivas (ROSS J, et al., 2007). 
Sendo assim o objetivo do trabalho foi discorrer e abordar através de uma revisão narrativa sobre manejo clínico de DIP/DIPA doença inflamatória pélvica de repetição.

\section{REVISÃO BIBLIOGRÁFICA}

A DIP é uma das doenças mais comuns encontradas em mulheres que têm relação sexual sem proteção. Segundo Taylor BD, et al. (2012), a DIP está interligada a consequências a longo prazo, o que acarreta problemas reprodutivos como infertilidade, gravidez ectópica e dor pélvica crônica em $18 \%$ dos casos. Sua prevalência é subestimada, pois a maioria dos casos é subclínica (>60\%) (JUDLIN PG, 2009).

As mulheres que já tiveram DIP têm chance de $12 \%$ a $15 \%$ de ter gravidez ectópica no futuro, e na questão da infertilidade a taxa é de $12 \%$ a $50 \%$ (CHEONG YC, et al., 2014). Estima-se um caso de DIP para cada 8 a 10 casos de pacientes com cérvice por algum desses patógenos. Toda mulher precisar ser acompanhada quando há indícios da DIP, principalmente quando possui situações em que o exame especular ou manual for realizado, quando pré-existir queixas de desconforto abdominal, dor dorsal, spotting ou dispareunia antecedendo os procedimentos transcervicais (DEKKER JH, et al., 2014). A maior prevalência é em mulheres ativas entre 15-24 anos de idade (LAREAU SM e BEIGI RH, 2008).

Em média $12 \%$ das adolescentes que possuem vida sexual ativa vai apresentar no mínimo um episódio de DIP antes de atingir os 20 anos de idade (ROSS J, et al., 2007). A partir das informações do Centers for Disease Control and Prevention (2007), outra forma de contrair a DIP é ter parceiros múltiplos e sexo sem prevenção contribuem para a contaminação, enquanto fazer o uso de preservativo diminui o risco da doença.

De acordo com Micaela B (2011), o diagnóstico da DIP deve sempre basear-se nos achados clínicos. Os exames complementares são pedidos para pacientes que ainda estão com diagnóstico incerto, cujo estado já é grave ou que não conseguem responder ao tratamento, e buscar eliminar outra possível patologia ou minimizar complicações graves da doença inflamatória pélvica. A doença inflamatória pélvica aguda é um problema de saúde pública, pelo fato de se associar a complicações médicas importantes (infertilidade, gravidez ectópica e dor pélvica crônica) assim como a cuidados de saúde de custos elevados. Daí a importância de um diagnóstico precoce e de estratégias de tratamento adequadas e de rápido início para prevenir ou até mesmo minimizar sequelas a longo prazo.

Halperin R, et al. (2003), em um estudo retrospectivo, relataram uma associação entre idade 'mais velha' e DIP complicada, não analisando o papel do uso do DIU, mesmo que de acordo com seus dados, $50 \%$ dos casos fossem usuários de DIU, sugeriram, no entanto, que havia uma associação entre a longa duração do uso do DIU e a gravidade da doença.

Já em seu estudo Ness RB, et al. (2004), abordaram 684 mulheres sexualmente ativas com doença inflamatória pélvica (DIP) que foram monitoradas por aproximadamente 35 meses, onde foram relacionados ao uso de contraceptivos à recorrência autorreferida de DIP, dor pélvica crônica e infertilidade. O uso de aplicação corretamente do uso de preservativo durante o tempo do estudo conseguiu reduzir o risco de DIP recorrente, dor pélvica crônica e infertilidade. O uso direto de preservativos (cerca de $60 \%$ dos encontros) na linha de base minimizou riscos, após ter sido feito regulagem para fatores de confusão, de $30 \%$ a $60 \%$. O uso correto e direto de auto relato do preservativo foi de grande valor para apresentar menores taxas de sequelas de DIP.

Edelman DA, et al. (1990), já traziam em seus estudos que a longa duração do uso do DIU parecia elevar o risco de DIP e que poderia acarretar complicações em seu curso clínico. Analisaram os riscos que poderiam aparecer por ter o uso prolongado do DIU em um artigo de revisão de estudos mais antigos e, com relação ao risco de DIP grave, afirmaram que alguns estudos demonstraram que o risco de DIP atribuído aumenta com a duração do uso do DIU.

O diagnóstico precoce é muito importante, pois não permite que a doença se agrave, sendo o tratamento ambulatorial indicado para as formas leves, e as internações hospitalares para tratamento via endovenosa (WISKE CP, 2016). 
No exame físico é importante verificar a temperatura axilar $>38^{\circ} \mathrm{C}$; ponderar o nível da dor no momento da palpação e também da descompressão brusca dolorosa no baixo ventre; dor ao toque do colo do útero e dos anexos; toque de tumor anexial doloroso (abscesso tubo-ovariano); convexidade dolorosa do fundo de saco vaginal (abscesso pélvico); canal cervical com corrimento branco, amarelado ou sangramento induzido (MARRAZZO JM, MARTIN DH, 2007)

Quanto aos exames laboratoriais são sugeridos quando é necessária uma investigação de DIP, e dentre os testes que são solicitados estão: teste de gravidez, exame a fresco da secreção vaginal, hemograma completo, teste para Clamídia e Gonococo, urocultura, proteína $C$ reativa, anti-HIV, além de ser importantes fazer a testagem para hepatite B e sífilis (SWEET RL, 2012).

O diagnóstico também pode ser enriquecido por exames de ultrassonografia pélvica transabdominal e transvaginal, além de tomografia computadorizada ou ressonância magnética, para ter uma melhor visualização do possível diagnóstico, devido à alta resolução que esses exames podem promover. Já quando relacionado a laparoscopia permite-se ter o diagnóstico mais preciso, uma vez que não se consegue comprovar a endometrite nem salpingite luminar (CENTERS FOR DISEASE CONTROL AND PREVENTION, 2007).

A ultrassonografia é o exame de imagem muito recomendado em pacientes com suspeito de abscesso, e podem encontrar achados que auxiliam o diagnóstico de DIP como coleções líquidas nas tubas uterinas e fundo de saco de Douglas, borramento nos contornos do útero e anexos e formações sólidas sugestivas de abscesso (VIECELLI CF, et al., 2003).

A prevenção das complicações agudas, assim como das sequelas em curto e a longo prazo, é um dos objetivos primordiais do tratamento da DIP (CAZORLA E, et al., 2005). A prevenção é fundamental para não adquirir as ISTS, e o principal método é o uso de preservativo na relação sexual (ROMANELLI RMC, et al., 2013).

Ainda segundo o autor Campos $O$, et al. (2009), recomenda-se que, sempre que existir suspeitas clínicas de DIP, é de boa conduta iniciar o tratamento nas primeiras 48 a 72 horas, mesmo que não consiga rastrear uma DST. Pois o tratamento correto e importante no tempo inicial sendo a melhor medida preventiva das complicações de DIP. A prevenção da DIP tem como objetivo primordial evitar a infertilidade e a intervenção deve assentar em dois pontos fundamentais: a nível dos fatores de risco e do tratamento.

Esta é melhor conseguida através da promoção da prevenção primária das DSTs as medidas preventivas a adotar são as relacionadas com o comportamento sexual, tais como iniciar mais tardiamente a atividade sexual, manter relações sexuais apenas com um parceiro saudável, também ele monogâmico, e usar o preservativo de forma correta e consistente, principalmente o masculino (CAMPOS O, 2009), e da detecção de início de infecções do trato genital inferior (HAGGERTY C e NESS R, 2006).

Segundo as Diretrizes de 2010 do Center for Disease Control and Prevention (CDC) o tratamento deve ser instituído frente a um quadro suspeito. A escolha dos antibióticos depende da disponibilidade, tolerância e juízo clínico, não havendo diferença na evolução dos casos de intensidade baixa ou moderada hospitalizados ou tratados em regime ambulatorial.

Os tratamentos de forma oral e parenteral são eficazes em método de clínica similar em pacientes com DIP leve ou moderada. (MITCHELL C e PRABHU M, 2013). O tratamento oral pode ser indicado e iniciado em mulheres que não precisam de internações mesmo apresentando com infecção leve a moderada, sendo os resultados clínicos apresentando mesma condições aos das pacientes que precisarão receber o tratamento parenteral. Desta forma se não houver resposta clínica ao tratamento oral em 72 horas, é importante que a paciente passe por uma nova avaliação para ter a confirmação do diagnóstico e se for necessário iniciar o tratamento de forma parenteral (QUINET BB, et al., 2012).

Já o tratamento parental em muitos casos é indicado para mulheres com DIP de categoria leve ou de moderada gravidade, uma vez que a terapia oral e parenteral pode apresentar eficácia similar. A passagem do tratamento oral para o parental pode ser iniciada com 24 a 48 horas o que será levado em consideração 
as condições da melhora clínica. Em mulheres com abscesso tubo-ovariano necessita ser realizada, pelo menos, 24 horas de acompanhamento e observação intra-hospitalar (CENTER OF DISEASE CONTROL AND PREVENTION- CDC, 2010).

Os tratamentos podem ser hospitalar quando é compreendido que a internação é indicada nos casos de salpingite complicada, abscesso tubo ovariano, peritonismo com sinais de sepse; em gestantes, na necessidade de excluir-se outros diagnósticos de emergência (como apendicite), na falha ou intolerância ao tratamento ambulatorial e em quadros onde a sintomatologia clinica indica gravidade febre alta, náuseas e vômitos importantes, dor abdominal severa (ROSS J, 2010).

Já quando o tratamento cirúrgico, indica-se a realização de laparoscopia ou laparotomia para tratamento de abscesso tubo-ovariano quando há a falha do tratamento clinico, quando ocorre a persistência ou aumento de massa pélvica apesar do tratamento com antibióticos, na piora clínica da paciente e quando se suspeita de rotura do abscesso, com sangramento intraperitoneal e quando o abscesso se localiza no fundo de saco de Douglas (SCHNEE DM, 2009).

Quando se escolhe o tratamento com antibiótico empírico procura-se ser estabelecido quando há suspeita do quadro, e visa cobrir ambos os microrganismos principais (gonococo e clamídia) assim como micoplasmas e bactérias aeróbicas e anaeróbicas presentes na vagina e endocervice (SWEET RL, 2011).

As pacientes quando iniciam o tratamento escolhido pelo seu médico começam a apresentar melhora clínica significativa ainda nos primeiros três dias, mas em alguns casos que não tenham evolução dos sintomas com diminuição de febre, dor e rechaço abdominal e redução da dor à mobilização uterina e de anexos nesse primeiros dias, comumente precisarão de hospitalização, acompanhado de uma nova sequências de exames para confirmar os diagnósticos onde em alguns casos inclui fazer laparoscopia diagnóstico, podendo ser preciso a intervenção cirúrgica (QUINET BB, et al., 2012).

De acordo com o Protocolo Clínico e Diretrizes Terapêuticas Infecções Sexualmente Transmissíveis da Comissão Nacional de Incorporação de Tecnologias no Sistema Único de Saúde (CONITEC, 2015). A melhora clínica das pacientes com DIP deverá acontecer nos três primeiros dias após o início do tratamento antimicrobiano. Se houver piora do quadro, considerar outros exames de imagem, como ressonância nuclear magnética ou tomografia computadorizada axial, para diagnósticos diferenciais ou complicações de DIP.

A paciente deverá retornar ao ambulatório para seguimento na primeira semana após a alta hospitalar e deverá seguir abstinência sexual até a cura clínica. As pacientes que usam DIU não precisam remover o dispositivo. Caso exista indicação, a remoção deve ser realizada somente após as duas primeiras doses do esquema terapêutico. Nesses casos, as pacientes devem ser orientadas sobre métodos alternativos de barreira, como diafragma, preservativo masculino e feminino. As duchas vaginais não estão recomendadas.

\section{CONSIDERAÇÕES FINAIS}

A DIP ainda é considerada como um problema de saúde pública, interligada a morbilidade na fase aguda, bem como a sequelas importantes a longo prazo, nomeadamente infertilidade tubar, gravidez ectópica e dor pélvica crônica. Em alguns casos o diagnóstico é confirmado por exame de ultrassonografia, e em algumas situações complementados por tomografia computadorizada (TC) e a Ressonância Magnética (RM). Por se tratar de uma doença de clínica com variações, e em algumas situações assintomática, preconiza a dificuldade de se diagnosticar no início, o que pode acarretar sequelas graves, sendo elas de natureza agudas como crônicas, em especial no período de constituição reprodutiva das pacientes, o que aumenta a atenção para o diagnóstico de DIP, conseguindo dar início a um tratamento empírico com antibióticos antecipando ou minimizando assim possíveis intercorrências, sendo em tempo oportuno.

\section{REFERÊNCIAS}

1. BRASIL. Ministério da Saúde. Manual de Controle das Doenças Sexualmente Transmissíveis - DST. Brasília: MS; jan.2006.

REAS/EJCH | Vol.12(10) | e5074 | DOI: https://doi.org/10.25248/reas.e5074.2020 
2. CAMPOS O, et al. Doença Inflamatória Pélvica. Manual de Ginecologia, Permanyer Portugal, 2009I: 185-193.

3. CAZORLA E, et al. "Enfermedad inflamatoria pélvica grave. Respuesta al ertapenem." Rev Esp Quimioterap 2005. 18(4): 331-334.

4. CENTER OF DISEASE CONTROL AND PREVENTION (CDC). Sexually transmitted diseases treatment guidelines. MMWR. 2010; 59 (RR-12):1-114.

5. CENTERS FOR DISEASE CONTROL AND PREVENTION. Updated recommended treatment regimens for gonococcal infections and associated conditions - United States, April 2007. Pelvic inflammatory disease (PID). Parenteral treatment.

6. CHEONG YC, et al. Non-surgical interventions for the management pelvic pain. Estados Unidos: The Cochrane Collaboration, 2014

7. DECHERNEY AH, et al. Current Diagnosis and Treatment - Obstetrics and Gyne- cology. Em: Ainbinder SW, Ramin Sm, DeCherney AH, eds. Sexually Transmitted Diseases \& Pelvic Infec- tions. 10.a ed. EUA: The McGraw Hill Companies; 2003. p. 673-89.

8. DEKKER JH, et al. Doença inflamatória pélvica. Resumo de diretriz NHG M50.2014.

9. EDELMAN DA, et al. When should intrauterine devices be removed and replaced? Br J Fam Plann 1990; $16: 132-8$.

10. HAGGERTY C, NESS R."Epidemiology, pathogenesis and treatment of pelvic inflammatory disease." Expert Rev. Anti Infect. Ther. 2006;4(2): 235-247.

11. HALPERIN R, et al. Abscesso tubovírus em mulheres mais velhas: a idade da mulher é um fator de risco para falha na resposta ao tratamento conservatory? Gynecol Obstet Invest 2003; 55: 211-5.

12. HODSON A. Pelvic inflammatory disease. Innov Ait. 2009; 2(9):517-21.

13. IBARROLA $M$, et al. "Patología infecciosa: vulvovaginitis, enfermedades de transmisión sexual, enfermedad inflamatoria pélvica, abscesos tubo ováricos." An. Sist. Sanit. Navar.2009 32(Supl. 1): 29-38

14. JUDLIN PG, et al. Physiopathologie, diagnostic et prise en charge des infections genitals hautes Pelvic inflammatory diseases. Gynecol Obstet Fertil. 2009;37(2):172-82

15. KHAN ZE, et al. Pelvic inflammatory disease and pelvic abscesses. Rev Gynecol Perinatal Practice 2006;6(3):185-91.

16. LAREAU SM e BEIGI RH. Pelvic Inflammatory Disease and Tubo- -ovarian Abscess. Infect Dis Clin N Am. 2008; 22:693-708.

17. MANAVI K. A review on infection with Chlamydia trachomatis. Best Pract Res Clin Obstet Gynaecol. 2006 Dec; 20(6):941-51

18. MARRAZZO JM, Martin DH. Management of women with cervicitis. Clin Infect Dis. 2007;44 Suppl 3: S102-10.

19. MICAELA B. Doença inflamatória pélvica aguda do diagnóstico ao tratamento, FMUC, 2011

20. MITCHELL C, PRABHU M. Pelvic inflammatory disease: current concepts in pathogenesis, diagnosis and treatment Infect Dis Clin North Am. 2013 Dec;27(4):793-809.

21. NESS RB, et al. A cluster analysis of bacterial vaginosis-associated microflora and pelvic inflammatory disease. Am $\mathrm{J}$ Epidemiol. 2005; 162:585-90.

22. NESS RB, et al. Avaliação por Pares. Pesquisa e Prática. American Journal of Public Health.Agosto de 2004; 94 : 1327-1329

23. PROTOCOLO CLÍNICO E DIRETRIZES TERAPÊUTICAS INFECÇÕES SEXUALMENTE TRANSMISSÍVEIS. Relatório de Recomendação. CONITEC Comissão Nacional de Incorporação de Tecnologias no Sistema Único de Saúde 2015.

24. QUINET BB, et al. Doença inflamatória pélvica: atualização. Rev Med Minas Gerais 2012; 22 (Supl 5): S50-S54

25. ROMANELLI RMC, et al. Abordagem atual da doença inflamatória pélvica. Revista Médica de Minas Gerais, Belo Horizonte, v. 23, n. 3, p.347-355, abr. 2013.

26. ROSS J, et al. European guideline for the management of pelvic inflammatory disease. Int J STD AIDS. 2007;18(10):662-6.

27. ROSS J. Pelvic Inflammatory Disease. Medicine. 2010;38(5):255-9.

28. SAMRAJ GP, et al. Acute pelvic pain: evaluation and management. Compr Ther. 2004;30: 173-84.

29. SCHNEE DM. Pelvic inflammatory disease. J Pedriatr Adolesc Gynecol.2009; 22:387-9.

30. SOUSA FB, et al. SISTEMA DE GESTÃO DA QUALIDADE. Protocolo Clínico. Doença inflamatória pélvica. Universidade Federal do Ceará, MEAC, EBESRH. 2018

31. SWEET RL. Treatment of Acute Pelvic Inflammatory Disease. Infect Dis Obstet Gynecol. 2011; 2011:56;1909.

32. SWEET RL. Treatment strategies for pelvic inflammatory disease. Expert Opin Pharmaco ther 2009;10(5): 823-837.

33. SWEET RL.Pelvic Inflammatory Disease: current concepts of diagnosis and management. Curr Infect Dis Rep. 2012; 14:194-203.

34. TAYLOR BD, et al.Racial Variation in Toll-like Receptor Variants Among Women With Pelvic Inflammatory Disease. The Journal of Infectious Diseases. Oxford, p. 940-944. agosto 2012.

35. TORTORA G, et al. Microbiologia. 8ํㅡㄹㅗão. Artmed Editora, 2005.

36. VIECELLI CF, et al. Doença inflamatória pélvica. Acta Médica 2003; 24:324-42.

37. WISKE CP, et al. Physician specialty influences care of pelvic inflammatory disease. Estado Unidos, mar. 2016. 